\title{
Comparative study analyzing survival and safety of bevacizumab/carboplatin/paclitaxel versus carboplatin/docetaxel in initial treatment of metastatic Her-2-negative breast cancer
}

\author{
This article was published in the following Dove Press journal: \\ Breast Cancer:Targets and Therapy \\ 16 June 2013 \\ Number of times this article has been viewed
}

\author{
Yasser Abdel Kader \\ Marc Spielmann² \\ Tamer El-Nahas' \\ Amr Sakr \\ Hassan Metwally ${ }^{3}$ \\ 'Department of Clinical Oncology, \\ Cairo University, Cairo, Egypt; \\ ${ }^{2}$ Department of Medical Oncology, \\ Institute Gustave Rousssy, VuilleJuif, \\ Paris, France; ${ }^{3}$ Department of Clinical \\ Oncology, Monufia University, \\ Monufia, Egypt
}

\begin{abstract}
Purpose: In view of the previous reports demonstrating the positive outcome of bevacizumab in metastatic breast cancer, we aimed at comparing the role of bevacizumab-based metronomic combination with taxane (paclitaxel) versus a different taxane (docetaxel)-based regimen in addition to carboplatin as initial treatment for metastatic Her-2-negative breast cancer.

Patients and methods: This is a randomized Phase III study comparing the progression-free survival (PFS) and safety in Her-2-negative female patients with initial diagnosis of metastatic breast cancer with World Health Organization performance status of 0 -II. Forty-one patients were randomized from September 2008 to July 2009 to receive either; (1) bevacizumab $5 \mathrm{mg} / \mathrm{kg}$ day 1 and day 15, carboplatin area under the curve (AUC)-2 day 1, day 8 , and day 15, and paclitaxel $60 \mathrm{mg} / \mathrm{m}^{2}$ day 1 , day 8, and day 15 (arm-I); or (2) carboplatin AUC-5 day 1, docetaxel $75 \mathrm{mg} / \mathrm{m}^{2}$ day 1 (arm-II). The Kaplan-Meier method was used for estimating survival; log-rank test for comparing survival curves. The primary end point was PFS, and secondary end points were overall survival (OS) and safety.

Results: PFS was 10 months in arm I versus 10.2 months in arm II $(P=0.9)$. The OS rate was similar in both arms: 37.6 months for arm I versus 37.4 months for arm II $(P=0.92)$. The toxicity revealed higher incidence of hypertension and proteinuria in arm I; however, with higher incidence of grade III-IV neutropenia and neutropenic fever in arm II. No treatment-related mortality was recorded.
\end{abstract}

Conclusion: Bevacizumab/carboplatin/paclitaxel and carboplatin/docetaxel show comparable PFS and OS with different toxicity profiles.

Keywords: taxanes, breast cancer, bevacizumab, Her-2 negative

\section{Introduction}

Breast cancer is the most common malignancy affecting women and the second cause of cancer death in the United States following bronchogenic carcinoma. ${ }^{1}$ A minority of patients initially present with metastatic breast cancer (MBC); however, it is estimated that $20 \%-30 \%$ of patients with early-stage disease will ultimately progress to metastatic disease. ${ }^{2}$ In this setting, including anthracyclines, taxanes and antimetabolites are the most preferred agents, but a single standard of care has not been identified. ${ }^{3}$ Actually, anthracycline-based regimens are commonly preferred in the treatment of metastatic disease, but due to cardiotoxicity, they have a limited role in patients previously exposed to anthracyclines in the adjuvant setting, hence the importance of developing new non-anthracycline regimens for the treatment of metastatic disease.
Correspondence: Tamer El-Nahas Department of Clinical Oncology, Cairo University, I Al Saray Street, Al Manial, Cairo, Egypt Fax +20 23347 7771

Email elnahas@sphinxcure.com 
Platinum complexes are active in a wide range of solid tumors. ${ }^{4}$ Although both cisplatin and carboplatin have shown activity in breast cancer, carboplatin appears as a more appropriate choice for treatment of metastatic disease, due to less severe non-hematologic toxicities.

Although no large Phase III trials are ongoing to compare carboplatin/paclitaxel combination therapy with paclitaxel alone for MBC, several Phase II studies have shown that combination therapy with carboplatin and paclitaxel is active and reasonably well tolerated as first-line treatment of patients with MBC. ${ }^{5}$ Weekly paclitaxel is active in patients with $\mathrm{MBC}$, especially patients treated previously in the adjuvant or metastatic setting with anthracyclines, and it has a mild toxicity profile. ${ }^{6}$ Moreover, some studies, such as the CALGB 9840 study, also demonstrated statistically significant improvements in response rate and time to progression, with a trend for improved survival using weekly instead of every 3 weeks (q3w) paclitaxel as first-line therapy for metastatic disease. ${ }^{7}$ Bevacizumab was previously tested in the setting of initial treatment of MBC; Eastern Cooperative Oncology Group (ECOG)-2100 randomized 680 previously untreated patients with MBC to paclitaxel $90 \mathrm{mg} / \mathrm{m}^{2}$ weekly, 3 out of 4 weeks, with or without bevacizumab $10 \mathrm{mg} / \mathrm{kg}$ given every 2 weeks. The combination yielded a superior response rate of $29.9 \%$ compared with $13.9 \%$ for single paclitaxel alone. Although progression-free survival (PFS) was superior to the combination (11.4 months compared with 6.11 months for paclitaxel; $P \leq 0.0001$ ), there was no difference in overall survival (OS) (28.4 months for the combination versus 25.2 months for paclitaxel alone) ${ }^{8}$ On the basis of this study, the Food and Drug Administration (FDA) gave the preliminary approval for the use of bevacizumab for MBC, and that was when our study was launched, prior to the withdrawal of bevacizumab by the FDA. Two subsequent studies, RIBBON $1^{9}$ and AVADO confirmed that the addition of bevacizumab improved response rate and PFS in MBC, but not OS. However, the absolute increases in PFS in RIBBON 1 and $\mathrm{AVADO}^{10}$ were not of the same magnitude as in ECOG-2100 (5.5 months in ECOG-2100 versus 0.9 in AVADO and 1.2 months for RIBBON I) ${ }^{11}$ As a result, ODAC (Oncology Drug Advisory Committee of the FDA) voted 12 to 1 to revoke the approval of bevacizumab for the treatment of MBC. This issue is still under review. ${ }^{12}$ However, in Europe, bevacizumab remains approved, only as a firstline therapy in combination with paclitaxel or capecitabine.

Carboplatin/docetaxel doublet has been also extensively studied in the setting of initial treatment of MBC. Data from Phase II trials has shown that the combination of carboplatin and docetaxel is active in the first-line treatment of MBC. The
North Central Cancer Treatment Group (NCCTG) investigated the role of carboplatin/docetaxel regimen in patients irrespective of Human Epidermal growth factor Receptor-2 (Her-2) status. ${ }^{13}$ The overall response rate reached $58 \%$, the median PFS time was 9.8 months, and the 1 -year survival rate was $72 \%$. Therefore, it was concluded that the combination of docetaxel and carboplatin showed activity in the first-line setting for $\mathrm{MBC}$ and that the toxicities of this regimen were acceptable.

In our developing country, the comparison between both regimens, bevacizumab/carboplatin/paclitaxel and carboplatin/docetaxel, was appealing due to the problematic financial burden of metronomic anti-angiogenic regimens adding to the cost of more frequent hospitalization days. We assumed that in view that weekly paclitaxel is more active than three weekly regimen in patients with $\mathrm{MBC},{ }^{6}$ yet, three weekly docetaxel is more active than weekly schedule, hence, was the treatment design. Besides, as anti-angiogenic treatment bevacizumab is better combined with paclitaxel, as in the ECOG-2100 study, rather than docetaxel, as in the AVADO trial. ${ }^{8,10}$ The bevacizumab was combined to paclitaxel (arm I), but with lower doses than usual (5 mg/kg every 2 weeks) to reduce expected major side effects and risks, which were contributing factors for its FDA withdrawal. This study was designed prior to the FDA withdrawal of bevacizumab in the setting of MBC; yet, even after its withdrawal this issue remains still under review, and in Europe, bevacizumab remains approved as a first-line therapy in combination with paclitaxel or capecitabine. Therefore, the study was considered logical to reach a comparative analysis of PFS and safety outcomes of both regimens.

\section{Patients and methods}

This is a randomized study comparing PFS and toxicity profiles of bevacizumab/carboplatin/paclitaxel versus carboplatin/ docetaxel in 41 females (age $\geq 18$ years) with HER-2-negative MBC with World Health Organization performance status (WHO PS) of 0 to II, who presented to Dar El-Fouad Hospital during the period from September 2008 to July 2009.

Forty-one patients were randomized in a 1:1 ratio to:

Arm I: Bevacizumab $5 \mathrm{mg} / \mathrm{kg} \rightarrow$ (Day [D]1 + D15).

Carboplatin area under the curve (AUC)-2 $\rightarrow$ (D1 + D8 + D15) .

Paclitaxel $60 \mathrm{mg} / \mathrm{m}^{2} \rightarrow(\mathrm{D} 1+\mathrm{D} 8+\mathrm{D} 15)$.

To be recycled every 28 days.

Arm II: Carboplatin AUC-5 $\rightarrow$ D1.

Docetaxel $75 \mathrm{mg} / \mathrm{m}^{2} \rightarrow \mathrm{D} 1$.

To be recycled every 21 days. 
Pretreatment assessment included medical history, physical examination, complete blood count/platelet, routine biochemical profile including CA15-3 assay. Metastatic workup included isotopic bone scan and computed tomography scans of the chest, abdomen, and pelvis. Patients with impaired hematological, hepatic, renal functions; or with central nervous system disease at presentation were excluded.

Clinical examination blood count, liver enzymes, and serum creatinine were performed on a 3-weekly basis, while CA15-3 was performed on a 6-weekly basis. Radiological images were performed following the third and sixth cycles of chemotherapy and by the end of the treatment course. Boneonly disease was evaluated by bone scan after 6 cycles.

Steroidal premedication started 24 hours prior to docetaxel and continued for 3 days, in addition to vigorous hydration and antiemetic for arm II. Twenty patients were assigned to arm I (bevacizumab/carboplatin/paclitaxel), and 21 patients were assigned to arm II (carboplatin/docetaxel).

The Kaplan-Meier estimator was used for estimating survival, and log-rank test was used for comparing survival curves. The primary end point of the study was PFS, while secondary end points were OS and safety.

\section{Results}

A total of 41 patients were randomly assigned (20 patients to bevacizumab/carboplatin/paclitaxel and 21 patients to carboplatin/docetaxel). The base line patients and disease characteristics are show in Table 1.

The median PFS for patients randomly assigned to (bevacizumab/carboplatin/paclitaxel) was 10 months and almost equivalent to that of (carboplatin/docetaxel) 10.2 months $(P=0.9)$. Figure 1 shows the Kaplan-Meier curve for PFS for both arms.

Table I Baseline patients and disease characteristics for randomly assigned patients

\begin{tabular}{llll}
\hline & \multicolumn{1}{c}{$\begin{array}{l}\text { Bevacizumab/ } \\
\text { carboplatin/paclitaxel }\end{array}$} & $\begin{array}{l}\text { Carboplatin/ } \\
\text { docetaxel }\end{array}$ & P-value \\
\hline Age (median) & $\begin{array}{l}50.6 \text { years } \\
\text { - Range }\end{array}$ & $\begin{array}{l}52.33 \text { years } \\
\text { 32-69 years }\end{array}$ & 0.59 \\
WHO PS & $37-69$ years & \\
- I & $17 / 20(85 \%)$ & $18 / 20(85.7 \%)$ & 0.66 \\
- II & $03 / 20(15 \%)$ & $03 / 21(14.3 \%)$ & \\
ER/PR status & & & \\
- Negative & $07 / 20(35 \%)$ & $06 / 21(28.6 \%)$ & 0.66 \\
- Positive & $13 / 20(65 \%)$ & $15 / 21(71.4 \%)$ & \\
Location of disease & & & \\
- Bone only & $03 / 20(15 \%)$ & $05 / 21(23.8 \%)$ & 0.697 \\
- Visceral & $17 / 20(85 \%)$ & $16 / 21(76.2 \%)$ & \\
\hline
\end{tabular}

Abbreviations: ER, estrogen receptor; PR, progesterone receptor; WHO PS, World Health Organization performance status.

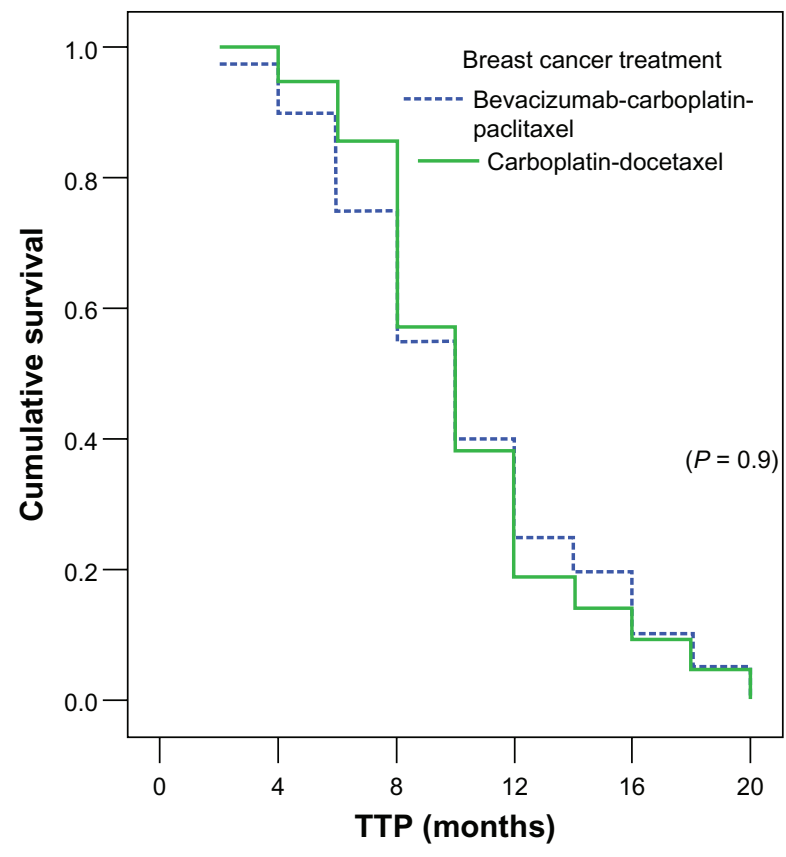

Figure I Median TTP in both arms.

Abbreviation: TTP, time to progression.

WHO PS was a strong prognostic factor for PFS; patients with PS 0-I had a PFS of 11 months, whereas patients with PS II had a PFS of 6 months $(P=0.035)$, as shown in Figure 2 .

It was also obvious that in the setting of Her- 2 negative status, estrogen receptor/progesterone receptor positive

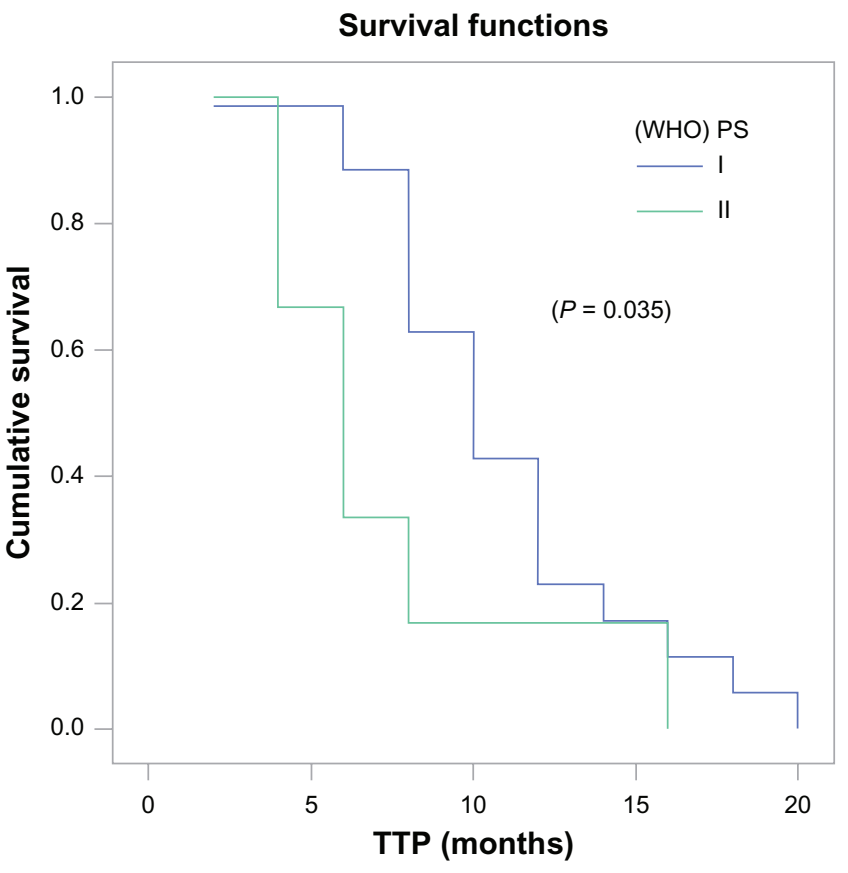

Figure 2 WHO PS correlation with TTP in the whole study group. Abbreviations: TTP, time to progression; WHO PS, World Health Organization performance status. 


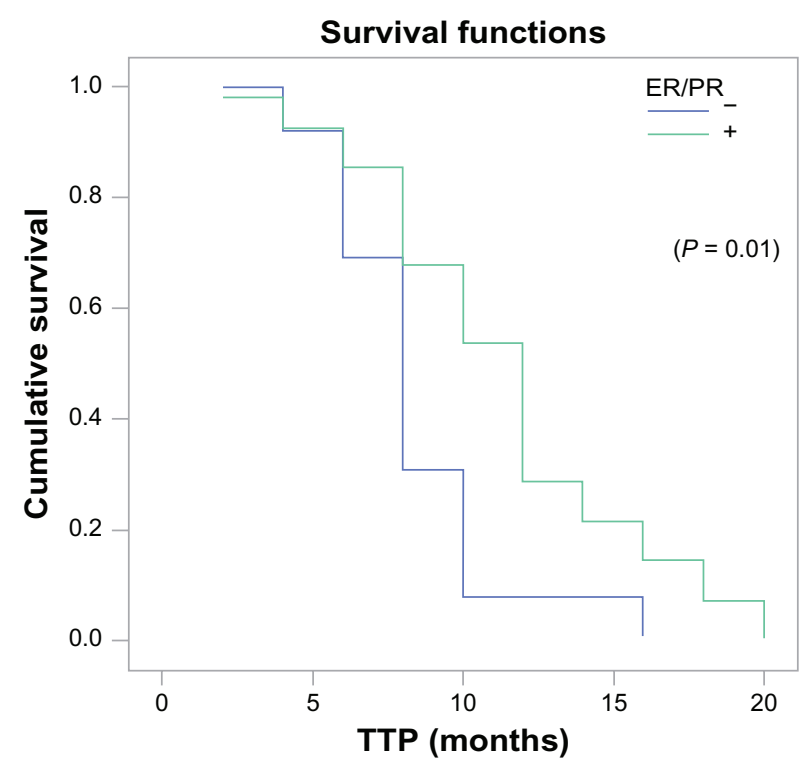

Figure 3 Steroid hormone correlation with TTP in the whole study group. Abbreviations: ER, estrogen receptor; PR, progesterone receptor; TTP, time to progression.

achieved better PFS of 12 months than triple negative, achieving median PFS of 8 months $(P=0.01)$ (Figure 3$)$.

Also, PFS was worse in patients with visceral metastasis (Figure 4), (10 months versus 12 months in patients with bone-only disease), but did not reach statistical significance $(P=0.872)$, and the median OS was numerically longer (Figure 5$)$ in the bone-only disease patients, (39.5 months versus 37.5 months in the visceral metastasis arm; $P=0.28$ ).

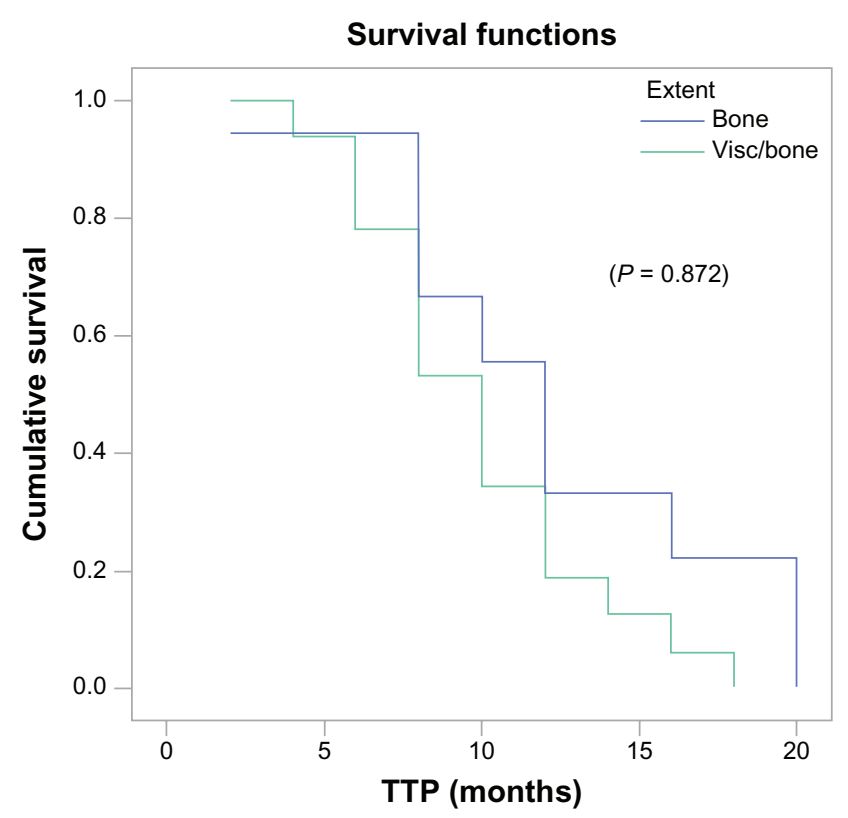

Figure 4 Bone/visceral metastasis correlation with TTP in the whole study group. Abbreviations: TTP, time to progression; visc, visceral.

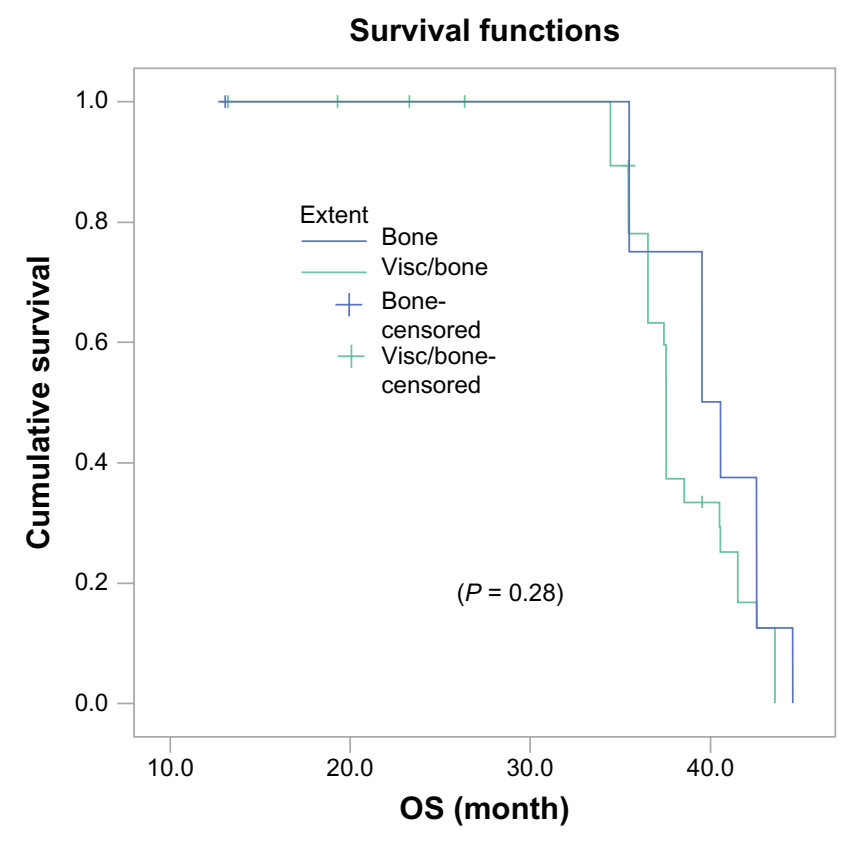

Figure 5 Bone/visceral metastasis correlation with median OS in the whole study group.

Abbreviation: OS, overall survival; visc, visceral.

The study did not show any difference in the median OS (Figure 6) for arm I (bevacizumab/carboplatin/paclitaxel), which was 37.6 months versus 37.4 months for (carboplatin/ docetaxel) $(P=0.92)$.

Subgroup analysis of patients less than 50 years (Figure 7) showed OS of 36.5 months, where patients over 50 years showed OS of 39.5 months.

\section{Safety}

The incidence of hypertension and proteinuria was higher in arm I, while neutropenia and neutropenic fever as well as

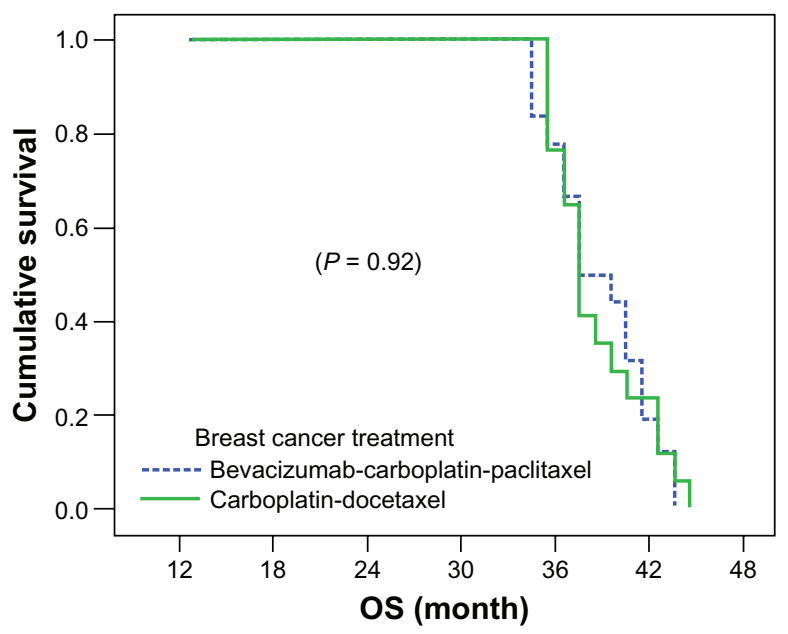

Figure 6 Median OS in both arms. Abbreviation: OS, overall survival. 


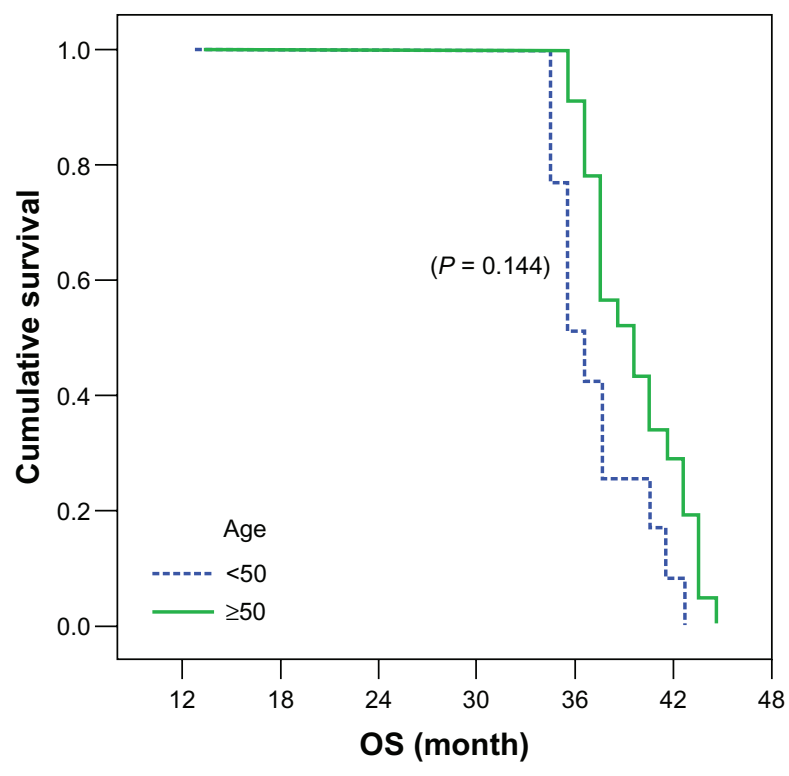

Figure 7 Age correlation with median OS in the whole study group. Abbreviation: OS, overall survival.

neuropathy were higher in arm II. However, deep venous thrombosis (DVT) was slightly higher in arm I, but did not reach statistical significance. No treatment-related mortality was recorded. Table 2 shows the encountered side effects evaluation in both arms.

\section{Discussion}

Due to cumulative cardiotoxicity and their common use in adjuvant chemotherapy, anthracycline-based regimens have a limited role in patients with MBC. Therefore, new nonanthracycline regimens are needed for metastatic disease. A considerable number of Phase II studies support incorporation of carboplatin as a standard agent in the management of patients eligible to receive first-line chemotherapy for MBC. Other Phase II studies of patients whose Her-2 status was unspecified demonstrated that the combination therapy with carboplatin and paclitaxel produced objective response rates of 53\%-62\%. ${ }^{5,14,15}$ These rates are substantially higher than those achieved in other Phase II trials of either single-agent

Table 2 Toxicity profile in both treatment arms

\begin{tabular}{llll}
\hline & $\begin{array}{l}\text { Bevacizumab/ } \\
\text { carboplatin/paclitaxel }\end{array}$ & $\begin{array}{l}\text { Carboplatin/ } \\
\text { docetaxel }\end{array}$ & P-value \\
\hline Hypertension & $04 / 20(20 \%)$ & $01 / 21(4.8 \%)$ & 0.18 \\
Proteinuria & $02 / 20(10 \%)$ & $00 / 21(0 \%)$ & 0.23 \\
DVT & $02 / 20(10 \%)$ & $01 / 21(4.8 \%)$ & 0.61 \\
Neutropenia & $04 / 20(20 \%)$ & $07 / 21(33.3 \%)$ & 0.34 \\
Neutropenic & $01 / 20(5 \%)$ & $04 / 21(19 \%)$ & 0.34 \\
fever & & $10 / 21(47.6 \%)$ & 0.41 \\
Neuropathy & $07 / 20(35 \%)$ & &
\end{tabular}

Abbreviation: DVT, deep vein thrombosis. carboplatin or paclitaxel. ${ }^{7,1718}$ The combination of carboplatin/ paclitaxel therapy was active in patients with anthracyclineresistant disease. ${ }^{10}$ Moreover, other studies pointed to synergy of bevacizumab with weekly paclitaxel over paclitaxel alone in improving PFS. ${ }^{8}$ Besides, results from Phase II studies also suggest that the combination of carboplatin and docetaxel is effective in the first-line treatment of metastatic disease $\mathrm{e}^{7,16}$ and comparable to previously mentioned studies. This study was designed to compare different taxanes schedules; carboplatin/docetaxel supported by the NCCTG-trial, ${ }^{13}$ with bevacizumab/carboplatin/paclitaxel supported by data from ECOG-2100 ${ }^{8}$, and was designed prior to bevacizumab FDA withdrawal. ${ }^{12}$

In our study, the median PFS for bevacizumab/carboplatin/ paclitaxel was 10 months, which was slightly less than the median PFS reported in ECOG-2100 of 11.8 months, while PFS in carboplatin/docetaxel was 10.2 months, which is almost the same as the median PFS reported in the NCCTGtrial of 9.8 months. The median PFS was similar in both arms; 10 versus 10.2 months $(P=0.9)$. The bevacizumab arm reported more toxicity as regards to hypertension and proteinuria in comparison with the non-bevacizumab arm. It is worth mentioning that in our study hypertension was recorded in $20 \%$ and proteinuria in $10 \%$ of patients in the bevacizumab arm versus $14.7 \%$ and $3.5 \%$ respectively in the E-2100 study, an incidence which was slightly higher but comparable with $14.7 \%$ for hypertension $3.5 \%$ in E-2100 study. The rate of DVT in the bevacizumab arm was $10 \%$ higher than that reported in ECOG-2100 at 2.1\%, with the difference probably attributable to our smaller sample size; also, there was no statistical significance between both arms in our study concerning DVT. Neuropathy was recorded in $35 \%$ of patients versus $23.5 \%$ in ECOG-2100. The rate of hematological toxicity and neutropenic fever was higher in the carboplatin/docetaxel arm in our study $(P=0.34)$. The OS was equivalent in our study in both arms (37.6 months for arm I versus 37.4 months for arm II), which was longer than that reported in ECOG-2100 (26.7 months median survival), probably due to a different patient population and to our smaller sample size. Finally, it was obvious that patients older than 50 years achieved better survival; however, this did not reach statistical significance $(P=0.144)$, and this limitation could be attributed to the small sample size recruited. Age might have shown significance in a larger sized population.

In conclusion, regimens, bevacizumab/carboplatin/ paclitaxel and carboplatin/docetaxel exhibit comparable PFS and OS but different toxicity profiles (hematological toxicity favored the metronomic arm, while hypertension, proteinuria, 
and DVT did not). Bevacizumab treatment required more hospital admission and more financial budget, which were not justified in this setting in terms of the non-superior clinical value witnessed in our study; however, it will be interesting in the future if different molecular markers will potentially guide the selection of patients who will most likely benefit from anti-angiogenic treatment in $\mathrm{MBC}$.

\section{Acknowledgments}

The authors would like to acknowledge all of the oncology team registrars and nurses at Dar Al Fouad Hospital who have made it possible to conduct this clinical trial. We also acknowledge the invaluable support received from Dr Nelly (head of Statistics Department in National Cancer Institute, Egypt) for her voluntary contribution for statistical analysis.

\section{Disclosure}

The authors declare no conflicts of interest.

\section{References}

1. American Cancer Society. Cancer Facts and Figures 2003. Atlanta, GA: American Cancer Society; 2003.

2. Mincey BA, Perez EA. Concise review for clinicians: advances in screening, diagnosis and treatment of breast cancer. Mayo Clin Proc. 2004;79: 810-816.

3. Carlson RW, Anderson BO, Bensinger W, et al. NCCN Practice Guidelines in Oncology, Version 2. National Comprehensive Cancer Network; 2002.

4. Go RS, Adjei AA. Review of the comparative pharmacology and clinical activity of cisplatin and carboplatin. J Clin Oncol. 1999;17:409-422.

5. Fountzilas G, Athanassiades A, Papadimitriou V, et al. Paclitaxel and carboplatin as first-line chemotherapy for advanced breast cancer. Oncology (Huntingt). 1998;12(Suppl 1):45-48.

6. Perez EA, Vogel CL, Irwin DH, et al. Multicenter Phase II trial of weekly paclitaxel in women with metastatic breast cancer. J Clin Oncol. 2001;19:4216-4223.
7. Seidman AD, Berry D, Cirrincione C, et al. Phase III weekly paclitaxel via 1 -hr infusion vs. standard 3 -hr infusion every third week in the treatment of metastatic breast cancer, with trastuzumab for HER2 ${ }^{+} \mathrm{MBC}$ and randomized to trastuzumab for HER2 normal MBC. Proc Am Soc Clin Oncol. 2004;23:6sa.

8. Miller KD. E2100: a phase III trial of paclitaxel versus paclitaxel/ bevacizumab for metastatic breast cancer. Clin Breast Cancer. 2003; $3(6): 421-422$.

9. Robert NJ, Diéras V, Glaspy J, et al, RIBBON-1: randomized, doubleblind, placebo-controlled, phase III trial of chemotherapy with or without bevacizumab for first-line treatment of human epidermal growth factor receptor 2-negative, locally recurrent or metastatic breast cancer. J Clin Oncol. 2011;29(10):1252-1260.

10. Pivot X, Schneeweiss A, Verma S, et al. Efficacy and safety of bevacizumab in combination with docetaxel for the first line treatment with elderly patients with locally recurrent or metastatic breast cancer: results from AVADO. Eur J Cancer. 2011;47:2387-2395.

11. Miles DW, Chan A, Dirix LY, et al. Phase III study of bevacizumab plus docetaxel compared with placebo plus docetaxel for the first line treatment of human epidermal growth factor receptor 2 negative metastatic breast cancer. J Clin Oncol. 2010;28(20):3239-3247.

12. Ocana A, Amir E, Vera F, et al. Addition of bevacizumab to chemotherapy for treatment of solid tumor; similar results but different conclusions. $J$ Clin Oncol. 2011;29(3):254-256.

13. Fitch RA, Suman VJ, Mailliard JA, et al. N9932: Phase II cooperative group trial of docetaxel (D) and carboplatin (CBDCA) as first-line chemotherapy for metastatic breast cancer (MBA). Proc Am Soc Clin Oncol. 2003;22:23.

14. Perez EA, Hillman DW, Stella PJ, et al. A Phase II study of paclitaxel plus carboplatin as first-line chemotherapy for women with metastatic breast carcinoma. Cancer. 2000;88:124-131.

15. Loesch D, Robert N, Asmar L, et al. Phase II multicenter trial of a weekly paclitaxel and carboplatin regimen in patients with advanced breast cancer. J Clin Oncol. 2002;20:3857-3864.

16. Brufsky A, Matin K, Cleary D, et al. A Phase II study of carboplatin and docetaxel as first line chemotherapy for metastatic breast cancer. Proc Am Soc Clin Oncol. 2002;21:52b.

17. Martin M, Diaz-Rubio E, Casado A, et al. Carboplatin: an active drug in metastatic breast cancer. J Clin Oncol. 1992;10:433-437.

18. O'Brien ME, Talbot DC, Smith IE. Carboplatin in the treatment of advanced breast cancer: a Phase II study using a pharmacokinetically guided dose schedule. J Clin Oncol. 1993;11:2112-2117.
Breast Cancer: Targets and Therapy

\section{Publish your work in this journal}

Breast Cancer: Targets and Therapy is an international, peerreviewed open access journal focusing on breast cancer research, identification of therapeutic targets and the optimal use of preventative and integrated treatment interventions to achieve improved outcomes, enhanced survival and quality of life for the cancer patient.

\section{Dovepress}

View the full aims and scopes of this journal here. The manuscript management system is completely online and includes a very quick and fair peer-review system, which is all easy to use. Visit http:// www.dovepress.com/testimonials.php to read real quotes from published authors. 